\title{
Dinâmica da composição botânica numa pastagem natural sob efeito de diferentes ofertas de forragem
}

\author{
Dynamic botanical composition in rangeland under forage allowances
}

\author{
André Brugnara Soares ${ }^{\mathrm{I}}$ Paulo César de Faccio CarvalhoII Carlos NabingerII \\ José Pedro Pereira Trindade ${ }^{\mathrm{III}}$ Júlio Kuhn Da Trindade ${ }^{\mathrm{IV}}$ Jean Carlos Mezzalira $^{\mathrm{IV}^{*}}$
}

\section{RESUMO}

\begin{abstract}
A intensidade de pastejo e suas alterações ao longo do tempo podem afetar a composição botânica e a estrutura do pasto. Este trabalho buscou avaliar o efeito de distintas ofertas de forragem (OF) sobre a dinâmica populacional de espécies de uma pastagem natural dos Campos Sulinos. As avaliações da composição botânica foram realizadas em 12 de janeiro e 17 de julho através do método BOTANAL. Os tratamentos constaram de diferentes OF $\left(\mathrm{kg} \mathrm{MS} 100 \mathrm{~kg}^{-1}\right.$ de peso vivo dia $\left.{ }^{-1}\right): 8,12$ e 16 durante todo ano e diferentes combinações de sequências: 8 na primavera e 12 no resto do ano (8-12); 12 na primavera e 8 no resto do ano (12-8); 16 na primavera e 12 no resto do ano (16-12). $O$ delineamento utilizado foi o de blocos casualizados com duas repetições. A oferta de forragem provocou alterações na composição botânica da vegetação. A manipulação da OF na primavera alterou a composição botânica da vegetação e apresentou maior impacto nas menores $O F$.
\end{abstract}

Palavras-chave: campo natural, composição botânica, variação da oferta de forragem.

\section{ABSTRACT}

Grazing intensity manipulation can affect structure and botanical composition of the pasture. The objective of this research was to evaluate the effects of different forage allowances and different seasonal sequences of forage allowance (FA) on the species population dynamics in the rangelands of Campos Sulinos. The botanical composition evaluations were made in January $12^{\text {th }}$ and July $17^{\text {th }}$, using BOTANAL method. Treatments were of different FA ( $\mathrm{kg} D M 100 \mathrm{~kg}^{-1}$ of live weight day $\left.^{-1}\right): 8,12,16$ all around the year; and different sequences of FA: 8 on spring and 12 at the rest of year (8-12); 12 on spring and 8 at the rest of year (12-8) and 16 on spring and 12 at the rest of year (16-12). The statistical design used was randomized blocks with two replications. The forage allowance led to changes in botanical and structural composition of vegetation. It can be inferred that the forage allowance variation on the vegetation had higher impact on smaller forage allowances.

Key words: botanical composition, rangeland, forage allowance variation.

\section{INTRODUÇÃO}

Os efeitos do pastejo sobre a dinâmica vegetal acontecem em diversos níveis dentro de uma escala de tempo. Em curto prazo, desfolhações diferentes, comandados pela preferência e seletividade animal, atrelados com a resistência de pastejo de cada espécie, podem mudar a composição botânica de uma pastagem (SCHÖNBACK et al., 2010). Em longo prazo, o pastejo modifica as propriedades físicas do solo, quantidade de nitrogênio no mantilho, perdas de nitrogênio do sistema, taxa de decomposição das raízes e do mantilho, que também fazem com que a composição botânica sofra ajustes (BIONDINI et al., 1998).

Essas interações modificam as relações competitivas intra e inter-específicas na comunidade

'Universidade Tecnológica Federal do Paraná (UTFPR), Pato Branco, PR, Brasil.

"II Universidade Federal do Rio Grande do Sul (UFRGS), Porto Alegre, RS, Brasil.

"IIEmpresa Brasileira de pesquisa agropecuária (EMBRAPA) Pecuária Sul, Bagé, RS, Brasil.

IVPrograma de Pós-graduação em Zootecnia, UFRGS, Porto Alegre, RS, Brasil. E-mail: mezzalirajc@gmail.com. *Autor para correspondência. 
e, ao longo do tempo, podem determinar mudanças florísticas perceptíveis. Com isso, as diferenças de intensidade de pastejo, que vêm sendo impostas na pastagem, poderiam ser avaliadas por meio da sua composição botânica. O presente trabalho teve por objetivo avaliar o efeito de diferentes ofertas de forragem sobre a dinâmica da composição botânica de uma pastagem nativa do Rio Grande do Sul mantida desde 1986 sob distintas OF.

\section{MATERIAL E MÉTODOS}

O trabalho foi conduzido em uma área de 52ha de pastagem natural da Depressão Central do Rio Grande do Sul (30'05'27' S e 51 $40^{\circ}$ ' $18^{\prime}$ 'O e altitude de $46 \mathrm{~m})$. O clima da região é do tipo Cfa, subtropical úmido com verão quente, segundo classificação de Köppen. A precipitação média anual é de $1440 \mathrm{~mm}$. O delineamento experimental utilizado foi o de blocos completamente casualizados, com duas repetições, sendo o tipo de solo usado como fator de bloqueamento. Sendo que um bloco ficou sobre um Argissolo vermelho distrófico típico e outro sobre um Plintossolo (EMBRAPA, 1999).

A área experimental vem sendo mantida desde 1986 com os mesmos tratamentos de $\mathrm{OF}(4,8,12$ e $16 \mathrm{~kg} \mathrm{MS} 100 \mathrm{~kg}^{-1}$ peso vivo dia $\left.{ }^{-1}\right)$, nas mesmas unidades experimentais (piquetes). Para este trabalho, cada piquete foi dividido em duas partes, sendo que uma parte continuou sendo manejada com a mesma $\mathrm{OF}$ e a outra teve sua $\mathrm{OF}$ aumentada ou diminuída conforme o tratamento. São estas: 8; 12; e 16 durante todo ano; 8 na primavera e 12 no verão, outono e inverno (8-12); 12 na primavera e 8 no verão, outono e inverno (12-8); e 16 na primavera e 12 no verão, outono e inverno. A oferta de forragem (OF) é expressa em kg MS $100 \mathrm{~kg}^{-1}$ peso vivo dia $^{-1}$. O método de pastejo foi o de lotação contínua com taxa de lotação variável visando a manter as OF preconizadas. O período experimental foi de 25/ $10 / 2000$ a 13/09/2001, compreendendo as quatro estações do ano (323 dias).

Para análise da composição botânica, fizeram-se duas avaliações, a primeira 22 dias após a mudança da OF nos devidos tratamentos, em 12 de janeiro de 2001, e a segunda, em 17 de julho de 2001. Foi utilizado o método Botanal (TOTHILL et al., 1992), realizando-se, em cada unidade experimental (piquete), 35 leituras (quadros de $0,25 \mathrm{~m}^{2}$ ) por avaliação. As espécies Andropogon lateralis e Aristida jubata foram classificadas em três níveis de pastejo: altamente pastejadas (AP), medianamente pastejadas (MP) e sem sinais evidentes de pastejo (BP), visando a estimar a contribuição, tanto em relação à massa de forragem, quanto sua contribuição na dieta animal, pois essas espécies apresentam-se entouceiradas a prostradas, como consequência das intensidades de pastejo.

Os dados foram analisados usando os softwares MULTIV (PILLAR, 1997) e SYNCSA (PILLAR \& ORLÓCI, 1993). Foram feitas análises de agrupamento tendo como critério a variância mínima, considerando-se os componentes com correlação aos eixos de ordenação superior a 0,6 . Os dados também foram submetidos à análise de ordenação por coordenadas principais. Outras informações sobre a metodologia deste trabalho podem ser obtidas em SOARES et al. (2005).

\section{RESULTADOS E DISCUSSÃO}

Nas duas avaliações realizadas na área experimental, foram registradas 126 espécies pertencentes a 26 famílias (SOARES, 2002). Na região da Serra do Sudeste, também no Rio Grande do Sul, CAPORAL \& BOLDRINI (2007) encontraram 173 espécies distribuídas em 34 famílias, o que indica a riqueza florística das diferentes formações campestres nativas.

A massa de forragem (MF - kg MS ha-1) de todas as espécies e do componente material morto foi considerada na análise multivariada dos dados e é o que comanda a dispersão dos tratamentos nos planos de ordenação (Figuras 1A e 2A). As figuras 1A e 1B representam a avaliação botânica dos piquetes avaliados em janeiro, sendo que a composição botânica é descrita pela MF das espécies componentes.

O diagrama de ordenação (Figura 1A) dos piquetes avaliados em 12 de janeiro representa $69,8 \%$ da variação total, sendo o eixo I (horizontal) responsável por 55\% dessa variação e o eixo II (vertical) por $14,8 \%$. Analisando-se o diagrama de ordenação, pode-se observar que, à medida que aumentam os valores do eixo I, aumenta a contribuição de Andropogon lateralis MP e diminui a de Paspalum notatum, Chevreulia acuminata, Piptochaetium montevidense, Eryngium horridum e Andropogon lateralis AP. No eixo II, à medida que aumentam os valores, aumenta a disponibilidade de Eryngium horridum.

Na figura 1B, observa-se a formação de três grupos de unidades experimentais por análise de agrupamento: grupo 1 - formado por um piquete do tratamento 16-12, outros dois piquetes, dos tratamentos 16 e 12-8; grupo 2 - formado pelos dois piquetes do tratamento $12 \mathrm{e}$ um piquete dos tratamentos 12-8, 16-12 e 16; grupo 3 - formado pelos dois piquetes do tratamento 8 e os dois piquetes do tratamento 8-12. Os tratamentos $16,16-12$ e $12-8$ apresentaram suas 


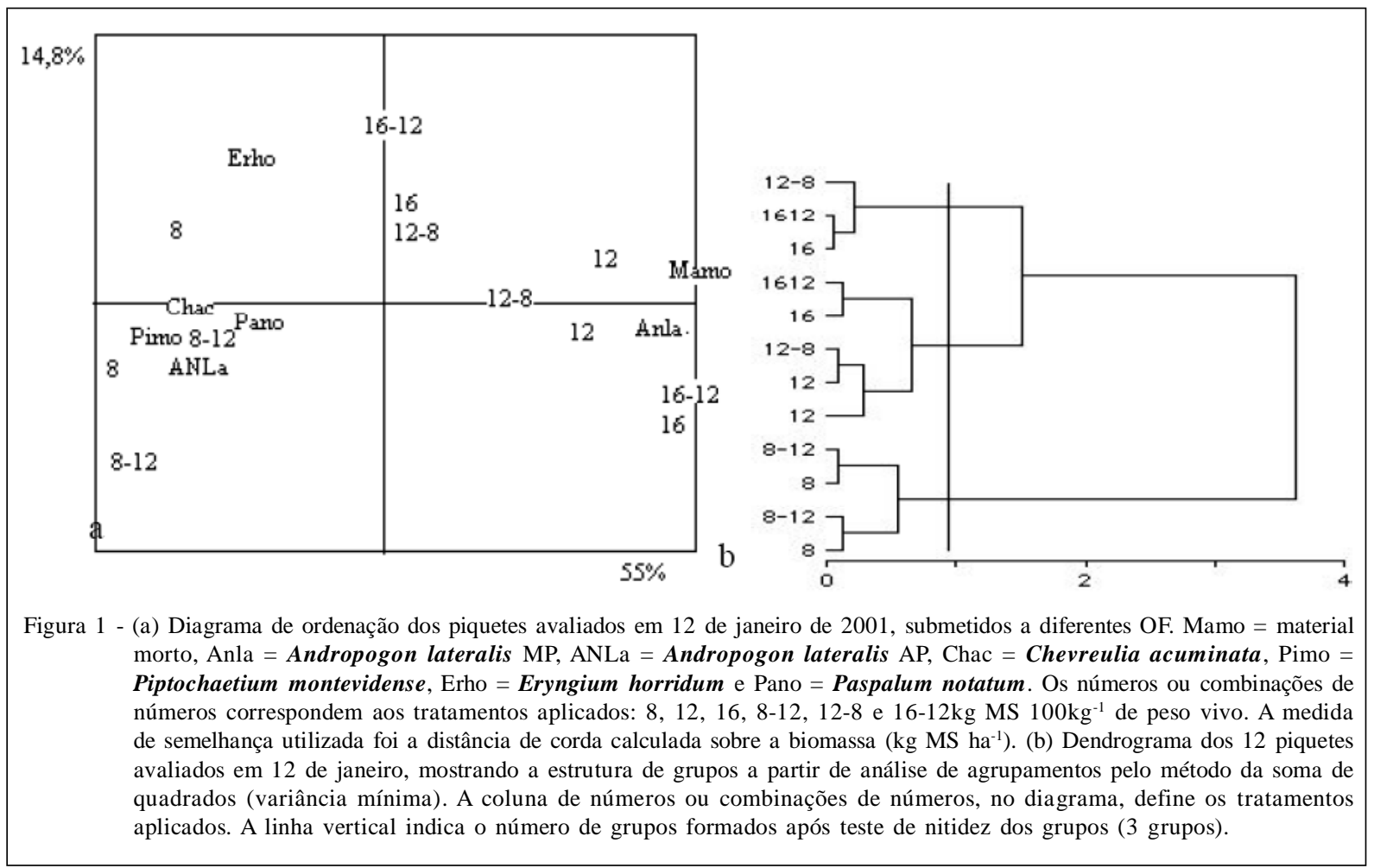

repetições em grupos diferentes, o que pode ser explicado pelo fato de cada repetição estar em um tipo de solo diferente.

O grupo 1 apresentou maior contribuição de Piptochaetium montevidense, Andropogon lateralis AP e Paspalum notatum. No grupo 2, ocorreu maior contribuição de Eryngium horridum, Hypoxis decumbens, Gamochaeta spp., Carex spp., Campomanesia aurea, Paspalum ionanthum, Panicum hians e Stylozanthes leiocarpa. Os piquetes que fazem parte do grupo 3 podem ser caracterizados por apresentarem uma maior contribuição de Andropogon lateralis MP, Aristida jubata MP e Schizachyrium microstachyum (Figuras 1A e 1B).

$\mathrm{Na}$ figura 2A, podemos observar o diagrama de ordenação dos piquetes avaliados no dia 17 de julho. Ele representa 49,2\% da variação total, sendo que $27 \%$ dessas variações são explicadas pelo eixo I (horizontal), e $22,2 \%$ pelo eixo II (vertical).

Na figura 2B, aparecem dois grupos de forma mais distinta. $\mathrm{O}$ grupo 1 é formado por cinco repetições (as duas do tratamento 12, as duas do tratamento $16 \mathrm{e}$ uma do 16-12), concentradas no quadrante superior direito da figura 2a e dominado por Andropogon lateralis MP. O grupo 2 é formado por sete repetições (as duas do 12-8, as duas do 8-12, as duas do 8 e uma do 16-12) e está localizado à esquerda do diagrama da figura 2A. Novamente, foi verificado maior grau de pastejo em Andropogon lateralis nas menores OF.

Houve alteração na composição e disponibilidade de espécies da primeira (12 de janeiro) para a segunda avaliação (17 de julho). Na Figura 3, encontra-se o diagrama de ordenação dos piquetes avaliados nos dias 12 de janeiro e 17 de julho. Os números correspondem aos respectivos tratamentos nas duas avaliações, 12 de janeiro (ponto de partida da seta) e 17 de julho (ponto de chegada da seta). As setas sugerem uma trajetória dos tratamentos entre as duas avaliações, indicando, dessa forma, uma mudança da composição botânica (dinâmica) dos piquetes em função das intensidades de pastejo.

Esse diagrama representa $61,1 \%$ da variação total, sendo que $34,7 \%$ são explicados pelo eixo I (horizontal) e $26,4 \%$ pelo eixo II (vertical). Os tratamentos 12 e 16 apresentaram trajetórias e direcionalidade semelhantes. Esses apontam para uma redução na disponibilidade das espécies Aristida jubata MP, Panicum hians, Andropogon lateralis MP e Briza subaristata, aumentando a disponibilidade das espécies Andropogon lateralis AP, Eryngium horridum e Axonopus sp. Essas mudanças estão na dependência da época do ano, uma vez que esses tratamentos foram de OF fixa. Nos tratamentos 8 e 8-12, pode-se observar decréscimo no componente 

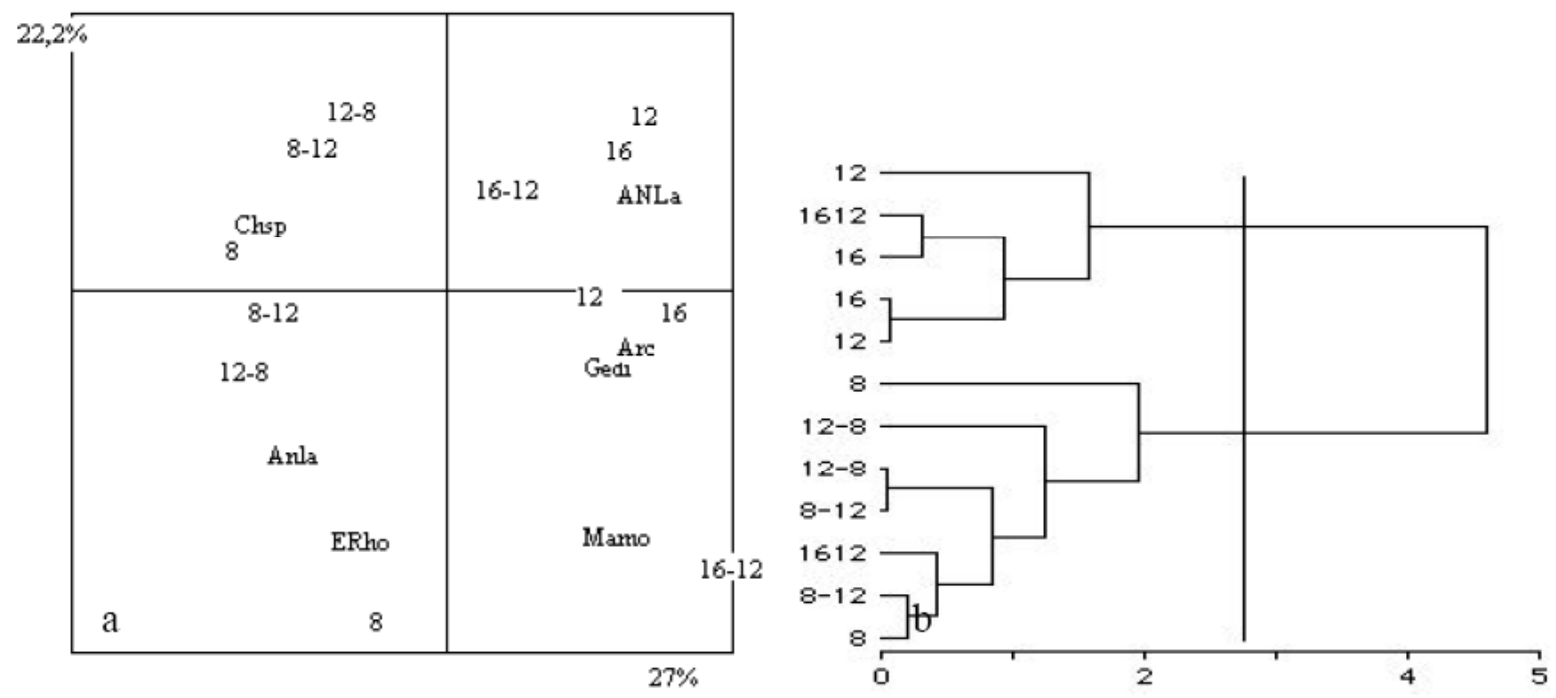

Figura 2 - (a) Diagrama de ordenação dos piquetes avaliados em 17 de julho de 2001, submetidos a diferentes OF. Mamo = material morto, Anla $=$ Andropogon lateralis $\mathrm{AP}, \mathrm{ANLa}=$ Andropogon lateralis $\mathrm{MP}, \mathrm{Chsp}=$ Chaptalia $\mathrm{sp}$. , Gedi $=$ Geranium dissectum, ERho $=$ Eryngium horridum e Arc $=$ Aristida jubata BP. Os números ou combinações de números correspondem aos tratamentos aplicados: $8,12,16,8-12,12-8$ e 16-12kg MS $100 \mathrm{~kg}^{-1}$ de peso vivo. A medida de semelhança utilizada foi a distância de corda calculada sobre a biomassa $\left(\mathrm{kg} \mathrm{MS} \mathrm{ha}^{-1}\right)$. (b) Dendrograma dos 12 piquetes avaliados em 17 de julho, mostrando a estrutura de grupos a partir de análise de agrupamentos pelo método da soma de quadrados (variância mínima). A coluna de números ou combinações de números, no diagrama, define os tratamentos aplicados. A linha vertical indica o número de grupos formados após teste de nitidez dos grupos (2 grupos).

Andropogon lateralis AP e, consequentemente, um ligeiro aumento da disponibilidade de Panicum hians, Andropogon lateralis MP, Briza subaristata, Aristida jubata MP, Eryngium horridum e Axonopus sp. Já o 16-12 teve menor alteração na sua composição e disponibilidade de forragem.

Os tratamentos manejados continuamente sob 8 e o tratamento de 8-12 apresentaram composições semelhantes nas duas avaliações, pelo qual se pode inferir que o impacto sobre a vegetação é maior ao se reduzir a oferta de 12 para 8 , comparado ao aumento de oferta de 8 para 12 . O tratamento $16-12$ e os tratamentos 12 e 16, na primeira avaliação, apresentaram características muito semelhantes (Figura 1B).

O dendograma da Figura 1B, que representa a primeira avaliação dos piquetes, indica que o grupo 3 é representado pelo agrupamento dos piquetes em que ocorreram as maiores intensidades de pastejo $(8,8-12$, 8, 8-12) e por isso predominou a presença de Paspalum notatum, de hábito prostrado, concordando com BOLDRINI \& MARASCHIN (1998), devido à sua excelente estratégia de proteção de gemas. No entanto, os referidos autores encontraram maior frequência de Andropogon lateralis em maiores $\mathrm{OF}$, enquanto que, neste trabalho, foi verificada alta frequência de Andropogon lateralis AP no grupo 3 da Figura 1B, que representa as maiores intensidades de pastejo, e formas do tipo MPe BP nas maiores OF. Provavelmente, essa diferença se deva à plasticidade fenotípica da espécie, o que permite que ocorra em diferentes intensidades de pastejo, e que ocorram em maiores frequências nos tratamentos de menores OF, pela sua tolerância ao pastejo.

Destaca-se, aqui, a importância da avaliação de graus de pastejo em espécies como Andropogon lateralis, já que se trata de uma espécie de alta plasticidade fenotípica (BOGGIANO, 2000). Sem essa distinção, provavelmente, os tratamentos de maiores e menores OF estariam agrupados. A espécie foi encontrada em todos os tratamentos, sendo sua presença pouco afetada pela intensidade do pastejo, influência apenas observada na sua estrutura. Com essa discriminação, foi possível verificar que, nos tratamentos de menor OF, Andropogon lateralis apresentou-se mais pastejada compondo o estrato inferior, ao passo que nas maiores OF aparecia no estrato superior, na forma de touceiras rejeitadas pelos animais.

Nos piquetes de maiores OF, representados pelo grupo 1 (Figura 1B), ocorre maior contribuição de formas entouceiradas de Andropogon lateralis.

O pastejo e especialmente a manutenção da mesma pressão de pastejo levaram a variações da composição florística da pastagem nativa, em 


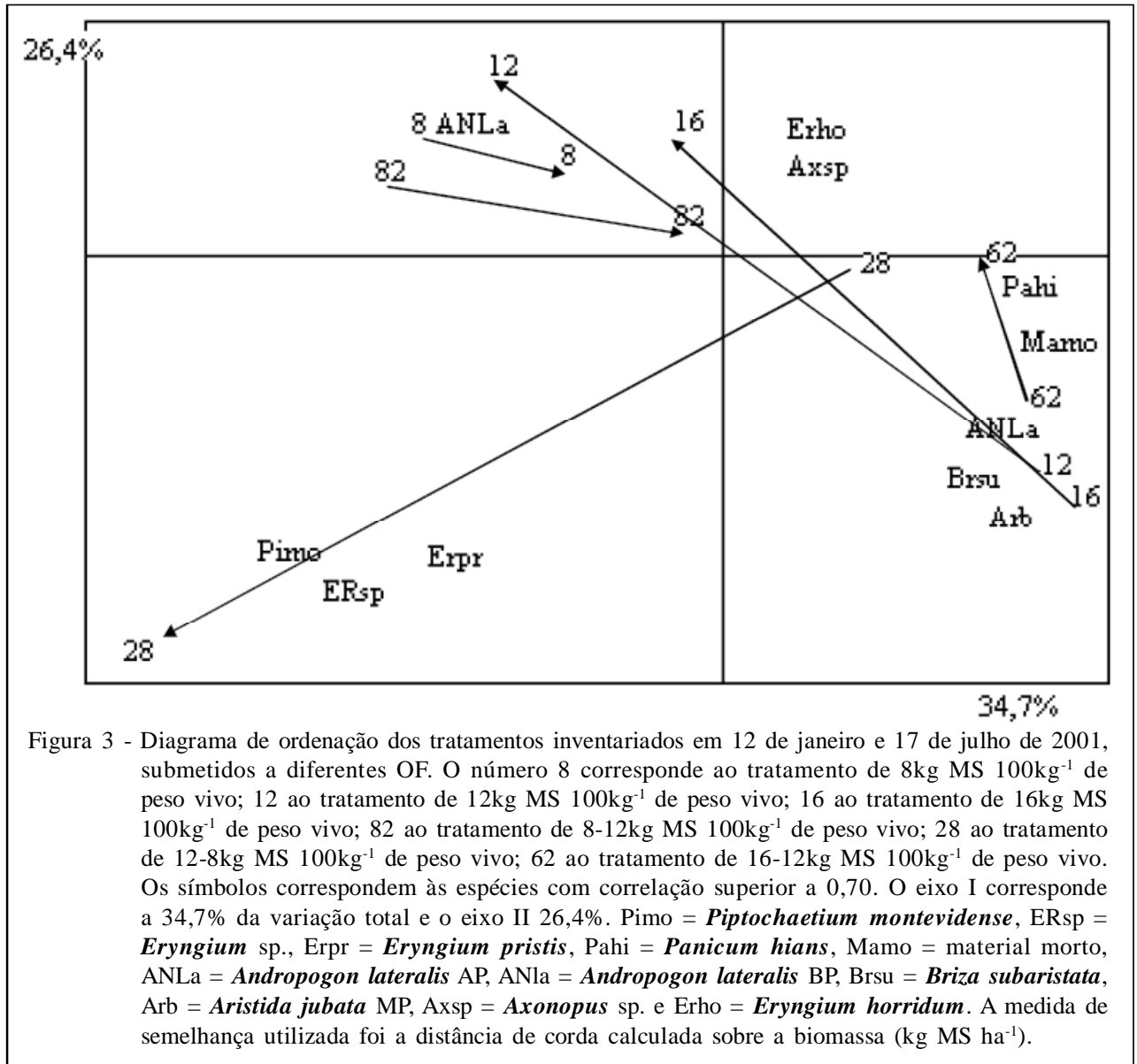

consonância com BIONDINI et al. (1998) que classificaram o pastejo como determinante da dinâmica da vegetação.

BOLDRINI \& MARASCHIN (1998) observaram que, em baixa intensidade de pastejo, tende a aumentar a presença de espécies como Eryngium horridum, Aristida jubata, Schizachyrium microstachyum. PILLAR et al. (1992) reportam que, em condições de baixa intensidade de pastejo, comunidades vegetais do tipo Desmodium - Axonopus - Paspalum tenderiam a evoluir para Baccharis Andropogon, enquanto que as do tipo Facelis Paspalum evoluiriam para comunidades com predominância de Eryngium sp.

Nesse contexto, o pastejo provoca alterações imediatas na composição botânica, fato observado no primeiro levantamento florístico realizado 22 dias após a alteração da OF. Foi possível observar esse efeito, principalmente, no tratamento em que a oferta foi diminuída de 12 para 8 . Conforme pode ser observado, a distância entre os tratamentos 8 ao 8-12 e 12 ao 12-8 foi maior do que a distância entre os tratamentos de 16-12 ao 16. Pode-se então inferir que o impacto da alteração da intensidade de pastejo sobre a vegetação de uma pastagem nativa é mais significativo nas menores OF.

Esse comportamento foi evidenciado estatisticamente (Figura 1B), pois, quando supostamente o tratamento de 12-8 deveria estar junto ao tratamento de 12 , devido a ter apenas 22 dias da mudança da OF, nota-se que uma repetição do 12-8 encontra-se em outro grupo, deslocando-se em sentido ascendente, diminuindo a proporção de Andropogon lateralis MP, Aristida jubata MPe material morto, além de aumentar a participação de Eryngium horridum. Ainda, observa-se que uma das repetições do tratamento 16-12 sempre ficou junto a uma repetição do tratamento 16 , indicando que a mudança de OF de 12 para 8 é diferente da mudança de 16 para 12.

Nota-se também que quando a OF foi diminuída de 12 para 8, a contribuição de Andropogon lateralis MP diminuiu, tornando as plantas que foram classificadas em MP (medianamente pastejadas) em AP (altamente pastejadas). Houve maior participação de espécies menos consumidas, como o Eryngium horridum. Esse aumento ocorreu, não pelo aumento 
de massa dessa espécie, e, sim, pela redução da massa das outras espécies, pois a redução da massa, com o aumento da pressão de pastejo, não ocorre, logicamente, de forma proporcional para todas as espécies, estando na dependência da seletividade das plantas pelos animais entre outros fatores.

Analisando as figuras 2A e 2B, que tratam da segunda avaliação botânica, nota-se novamente maior grau de pastejo em Andropogon lateralis nas menores OF, pois a espécie "ANLa", que é o $A$. lateralis medianamente pastejado, encontra-se bem mais perto dos tratamentos de alta OF que a "Anla", que representa o A. lateralis altamente pastejado. Trata-se de uma espécie potencialmente formadora de touceiras (SOARES, 2002), mas que se apresenta fazendo parte do estrato inferior juntamente com Paspalum notatum e Axonopus affinis nas menores OF, reafirmando sua habilidade adaptativa diante do distúrbio ocasionado pelo pastejo.

Analisando a figura 3, que trata das mudanças ocorridas na composição botânica do primeiro para o segundo levantamento, destaca-se o tratamento 12-8 (28) que teve sua composição drasticamente alterada, devido ao aumento na contribuição de Piptochaetium montevidense, Eryngium spp. e Eryngium pristis. Corroborando, dessa forma, FONTOURA Jr et al. (2004), que verificaram aumento de Eryngium horridum em função do aumento da intensidade de pastejo, fato que os autores atribuíram à pressão de pastejo. A maior contribuição dessas espécies, em relação ao levantamento anterior, pode ser atribuída ao aumento da pressão de pastejo e abertura da comunidade para o crescimento, especialmente Piptochaetium montevidense, e a redução de Andropogon lateralis MP (Figura 3).

Destaca-se também na dinâmica do tratamento 12-8, o qual teve redução na disponibilidade de espécies como Panicum hians, Andropogon lateralis MP, Centella asiatica, Briza subaristata, Aristida jubata MP, Panicum sabulorum, Schizachyrium microstachyum, Eryngium horridum, Baccharis trimera, Axonopus spp., Sporobolus indicus, Paspalum notatum e Desmodium incanum e aumento na disponibilidade das espécies Piptochaetium montevidense, Eryngium sp., Paspalum paucifolium, Eryngium pristis e Leptocoryphium lanatum. Explicam-se esses fatos pela abertura da comunidade, o que proporciona o desenvolvimento de espécies oportunistas e também, por consequência, do aumento da intensidade de pastejo, não somente pela redução do valor da $\mathrm{OF}$, mas também devido à seletividade animal, maior frequência de pastejo nas espécies preferidas em detrimento das indesejáveis.

Ainda analisando a dinâmica da vegetação (Figura 3), observa-se que, neste intervalo de tempo, janeiro a julho, a alteração da OF de 16-12 apresentou menor impacto sobre a composição da vegetação do que a variação de 12-8 de $\mathrm{OF}$, pois esses dois tratamentos, embora com a mesma magnitude de variação da oferta, tiveram comprimentos e direções de mudanças bem distintas. Para diminuir a OF de 16 para 12, foi necessária a utilização de maior carga animal/ ha do que para diminuir de 12 para 8 . Mesmo assim, a alteração da composição botânica e sua estrutura é menos evidente, provavelmente pela estrutura bimodal dos piquetes que vinham sendo historicamente manejados com alta $\mathrm{OF}$, e que a ação dos animais sobre esse estrato de touceiras é modesta. Essa evidência encontra respaldo nos resultados de CASTILHOS et al. (2007) que afirmam que em condições de menor OF é que se observam possíveis efeitos negativos do pastejo sobre a biodiversidade e a sustentabilidade do ecossistema.

Analisando os dois levantamentos (Figura 1A, 1B, 2A e 2B), observa-se que as espécies Andropogon lateralis, quando avaliado de acordo com seu grau de pastejo, e Eryngium horridum sempre aparecem como definidoras das diferenças entre os distintos manejos de intensidade de pastejo, indicando que essas espécies podem ser avaliadas para inferir sobre intensidade de pastejo desse tipo de pastagem natural.

\section{CONCLUSÃO}

As diferentes $\mathrm{OF}$ e suas alterações ao longo do ano são procedimentos eficazes de manipulação da estrutura e da composição botânica da pastagem natural. A mudança no valor da OF como critério definidor de intensidade de pastejo apresenta impactos diferentes se a alteração for no sentido ascendente ou descendente. Se a OF for diminuída, a composição botânica da pastagem é alterada mais rapidamente do que se fosse aumentada, mesmo que seja na mesma magnitude de troca. Quando a OF é diminuída, o impacto maior sobre a vegetação existe se a OF inicial for intermediária e não alta. Isso indica a importância da intensidade de pastejo como causador de distúrbios na comunidade vegetal pastoril. A avaliação da participação das espécies Andropogon lateralis, incluindo o seu grau de pastejo, e Eryngium horridum é um bom indicador para inferir sobre a intensidade de pastejo da pastagem natural. 


\section{REFERÊNCIAS}

BIONDINI, M.E. et al. Grazing intensity and ecosystem process in a northern mixed-grass prairie, USA. Ecological Applications, v.8, n.2, p.469-479, 1998.

BOLDRINI, I.I.; MARASCHIN, G.E. Efeito do pastejo e do solo sobre formas biológicas. Serie Técnica, 94, v.94, p.141144, 1998.

CAPORAL, F.J.M.; BOLDRINI, I. Florística e fitossociologia em um campo manejado na serra do sudeste, Rio Grande do Sul. Revista Brasileira de Biociência, v.5, p.2-3, 2007.

CASTILHOS, Z.M.S. et al. Composição florística de campo nativo sob diferentes ofertas de forragem. Revista Brasileira de Biociência, v.5, p.84-86, 2007.

EMPRESA BRASILEIRA DE PESQUISA AGROPECUÁRIA EMBRAPA. Centro Nacional de Pesquisa do Solo - CNPS. Sistema Brasileiro de Classificação de solos. Rio de Janeiro, 1999. 412p.

FONTOURA JUNIOR, J.A. et al. Dinâmica da vegetação em pastagem nativa da Serra do Sudeste do RS, submetida a métodos físicos e químicos para o controle de plantas indesejáveis, associados à intensidades de pastejo. In: REUNIÃO ANUAL DA SOCIEDADE BRASILEIRA DE ZOOTECNIA, 2004, Campo Grande, MS. Anais... Campo Grande: SBZ, 2004. 262p. p. $1-4$.

BOGGIANO, P.R. Dinâmica da produção primária da pastagem nativa em área de fertilidade corrigida sob efeito de adubação nitrogenada e oferta de matéria seca. 2000. 191f. Tese (Doutorado Plantas Forrageiras) - Programa de Pós-graduação em Zootecnia, Faculdade de Agronomia, Universidade Federal do Rio Grande do Sul, Porto Alegre, RS.

PILLAR, V.D. et al. Fatores de ambiente relacionados à variação vegetação de um campo natural. Pesquisa Agropecuária Brasileira, v.27, p.1089-1101, 1992.

PILLAR, V.P.; ORLÓCI, L. Character-based community analysis: the theory and an application program. Holanda, The Hague: SPB Academic, 1993.

PILLAR, V.P. Multivariate exploratory analysis and randomization testing with MULTIV. Coenoses, v.12, p.145148, 1997.

SCHÖNBACK, P. et al. Grassland responses to grazing: effects of grazing intensity and management system in an Inner Mongolian steppe ecosystem. Plant and Soil, v.340, p.103115,2010 .

SOARES, A.B. Efeito da alteração da oferta de matéria seca de uma pastagem natural sobre a produção animal e a dinâmica da vegetação. 2002. 187f. Tese (Doutorado Zootecnia) - Programa de Pós-graduação em Zootecnia, Faculdade de Agronomia, Universidade Federal do Rio Grande do Sul, Porto Alegre, RS.

SOARES, A.B. et al. Produção animal e de forragem em pastagem nativa submetida a distintas ofertas de forragem. Ciência Rural, v.35, n.5, p.1148-1154, 2005.

TOTHILL, J.C. et al. BOTANAL - A comprehensive sampling and computing procedure for estimating pasture yield and composition. 1. Field sampling. Tropical Agronomy, v.78, 24p. 1992. 